ARTIGO DE OPINIÃO

\title{
LEPTOSPIROSE E ENCHENTES:
}

UMA FALSA CORRELAÇÃO?

\author{
Fernando Dias de Avila-Pires ${ }^{1}$
}

\section{RESUMO}

Admite-se, geralmente, que ratos se dispersam com as enchentes e que as bactérias são disseminadas por sua urina. Um estudo retrospectivo de casos humanos de leptospirose ocorridos entre $1991 \mathrm{e}$ 1996, no estado de Santa Catarina e na grande Florianópolis, Brasil, confirmados por sorologia, correlaciona a incidência de chuvas com os casos humanos de leptospirose. Entretanto, uma análise mais detalhada dos dados primários sugere uma hipótese alternativa que dependerá de confirmação futura. A correlação entre enchentes e surtos epidêmicos poderia ser espúria, como sugere a análise da coincidência desses eventos, naquele período, com os dados sobre casos notificados, solicitações de exames e mortalidade.

DESCRITORES: Leptospirose. Enchentes. Epidemias. Correlação.

No dia 9 de novembro de 1995, circulou uma nota assinada por Paul N. Levett, diretor do UK MRC Leptospira Laboratory, em Barbados, relatando a ocorrência de casos fatais de leptospirose, concomitante com um grande surto de dengue tipo 1. O diretor chama a atenção para o seguinte fato: "Our feeling from clinical observations and from the serological results, is that the population have been so primed to recognize dengue that they are staying at home with lepto until the disease is so advanced that it may be too late to do very much".

A correlação da ocorrência de casos humanos de leptospirose com chuvas fortes e enchentes é responsável por diagnósticos tardios nas épocas de estiagem e por uma falsa concepção da epidemiologia e ecologia da doença. Assim, Barcellos e Sabrosa (2001), analisando um surto ocorrido em 1996, no Rio de Janeiro, enfatizam: "A combination of solid waste accumulation and flood conditions is the most appropriate theoretical explanation for leptospirosis". Adiante, ressaltam o

1 Departamento de Medicina Tropical, Instituto Oswaldo Cruz, Rio de Janeiro, Brasil.

Endereço para correspondência: Fernando Dias de Avila-Pires, Departamento de Medicina Tropical, Instituto Oswaldo Cruz, Av. Brasil 4365, CEP 21045-900, Rio de Janeiro, Brasil. E-mail: favila@matrix.com.br

Recebido para publicação em 15/3/2006. Revisto em 5/10/2006. Aceito em 2/11/2006. 
risco da presença de roedores. Da mesma forma, segundo Pollack (1999) a doença é caracterizada como "A disease transmitted by water contaminated with rat urine".

Por outro lado, enquanto ratos, subentendendo-se aqui as espécies cosmopolitas e urbanas ou ruderais do gênero Rattus, são considerados geralmente como responsáveis pelos surtos epidêmicos, existem abundantes evidências que apontam para mecanismos diversos de contaminação e de transmissão direta e indireta, envolvendo um elevado número de hospedeiros e reservatórios não humanos (Collares-Pereira et al., 1997; Collares-Pereira et al., 2000; Ciceron et al., 2000; Michel et al., 2001).

A explicação corrente, tanto entre leigos como entre epidemiólogos, é que ratos se dispersam com as enchentes e as bactérias são disseminadas por sua urina. No entanto, a correlação entre enchentes e surtos epidêmicos parece ser espúria, como demonstra a análise da coincidência desses eventos realizada em Florianópolis, Santa Catarina, Brasil, cobrindo o período de janeiro de 1991 a abril de 1996, com os dados sobre casos notificados, solicitações de exames e mortes ocorridas naquele período (Kupek e Faversani, 2000).

O estudo revelou que houve coincidência entre três picos de incidência de leptospirose e os picos de precipitação atmosférica verificados nos anos de 1991, 1994 e 1996, o que, aparentemente, confirmaria a correlação propalada. Contudo, o número de requisições de exames ao Laboratório Central reduziu-se em 1992 e 1993, anos em que não houve enchentes. Em 1993, ano em que não houve cheia, o número de exames realizados foi menor; a taxa de resultados positivos foi semelhante à dos anos de enchentes, mas a letalidade foi maior do que em anos de cheia.

Os dados sugerem que, nas épocas de enchentes, já são esperados os casos de leptospirose, portanto há maior número de diagnósticos preliminares corretos e de solicitações de exames. Nos anos em que não há cheia, o diagnóstico é feito tardiamente e cresce o número de óbitos.

Os surtos epidêmicos de leptospirose raramente são investigados em profundidade, possivelmente por ser uma infecção de distribuição cosmopolita e relativamente comum. No Brasil, foram registrados 53.400 casos entre 1986 e 2003. Seus diversos nomes populares indicam as fontes mais conhecidas de infecção: doença do rato, doença dos porqueiros, febre dos arrozais, febre dos canaviais, doença dos cães (Acha e Szyfres, 1986). Nas áreas urbanas, limpadores de caixas-d'água, de fossas e valas constituem grupos de maior risco; nas zonas rurais, o contato direto com urina de animais silvestres, comensais e domésticos é um importante fator de infecção. Entretanto, abundam na literatura relatos de que a fonte de infecção não foi identificada. Os diversos sorovares de Leptospira spp têm como reservatórios, em sua ampla área de ocorrência, mamíferos domésticos e silvestres, insetívoros, suídeos, bovídeos, cervídeos, equídeos, canídeos, felídeos, além de murídeos.

Os sintomas, na fase inicial, podem se confundir com os de influenza, infecções pulmonares diversas, dengue, infecções renais e doenças ictéricas. 
O costume de se atribuir os surtos epidêmicos a enchentes e ratos esconde, na verdade, cadeias ecológicas mais complexas. Se, por um lado, enchentes colocam pessoas e hospedeiros não humanos em maior contato e ajudam a disseminar urina contaminada, por outro, tendem a reduzir os inóculos, o que pode contribuir para a ocorrência de infecções menos graves e para a redução da letalidade. A identificação de diversos sorovares em uma única enchente indica a contribuição de diferentes reservatórios, como aconteceu durante a epidemia ocorrida na Nicarágua, mencionada por Carole Bolin (1998), na qual cães desempenharam papel importante.

Em 1998, o fenômeno El Niño também foi invocado para explicar a epidemia que ocorreu no Equador, como também o foi em outros países para desviar a atenção da falta de ações de controle de epidemias, como a de dengue no Brasil.

Um estudo retrospectivo de casos humanos de leptospirose, confirmados por sorologia, ocorridos entre 1991 e 1996 no estado de Santa Catarina e na grande Florianópolis, correlaciona a incidência de chuvas com os casos de leptospirose (Kupek e Faversani, 2000). Entretanto, uma análise mais detalhada dos dados primários (Faversani et al., 1999) pode levar a uma interpretação diferente.

À primeira vista, verifica-se uma coincidência das curvas de pluviosidade máxima e total com os casos confirmados de leptospirose no período considerado (Figura 1 e Figura 2).

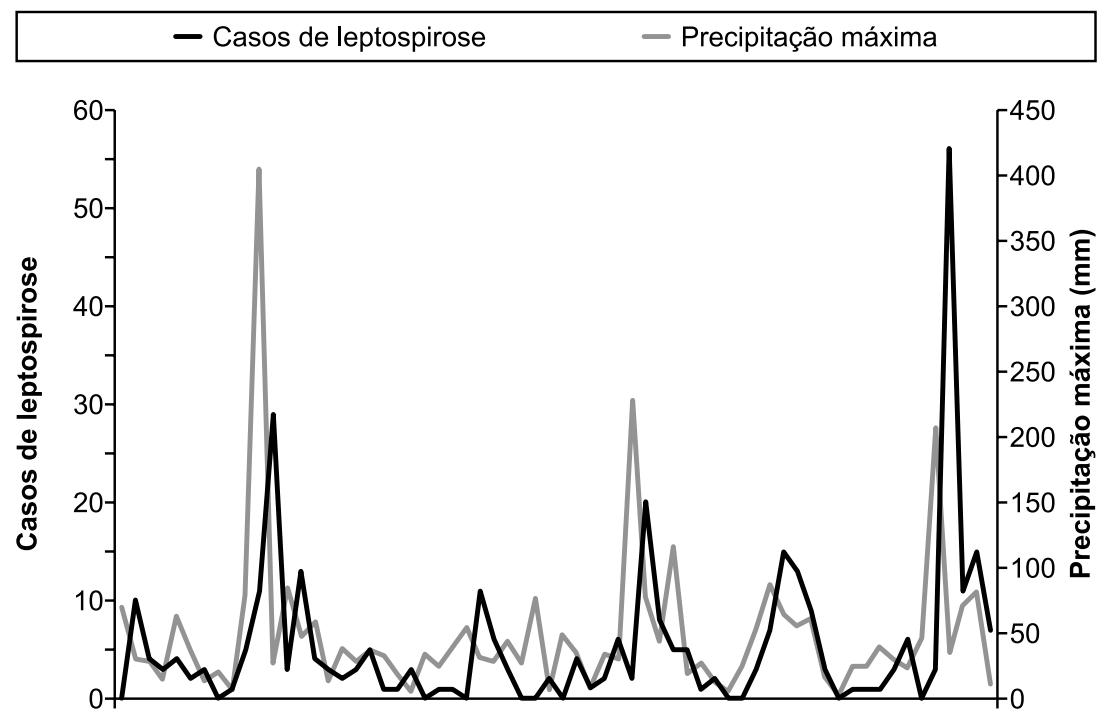

Figura 1. Casos de leptospirose confirmados pelo LACEN e precipitação pluviométrica máxima. 


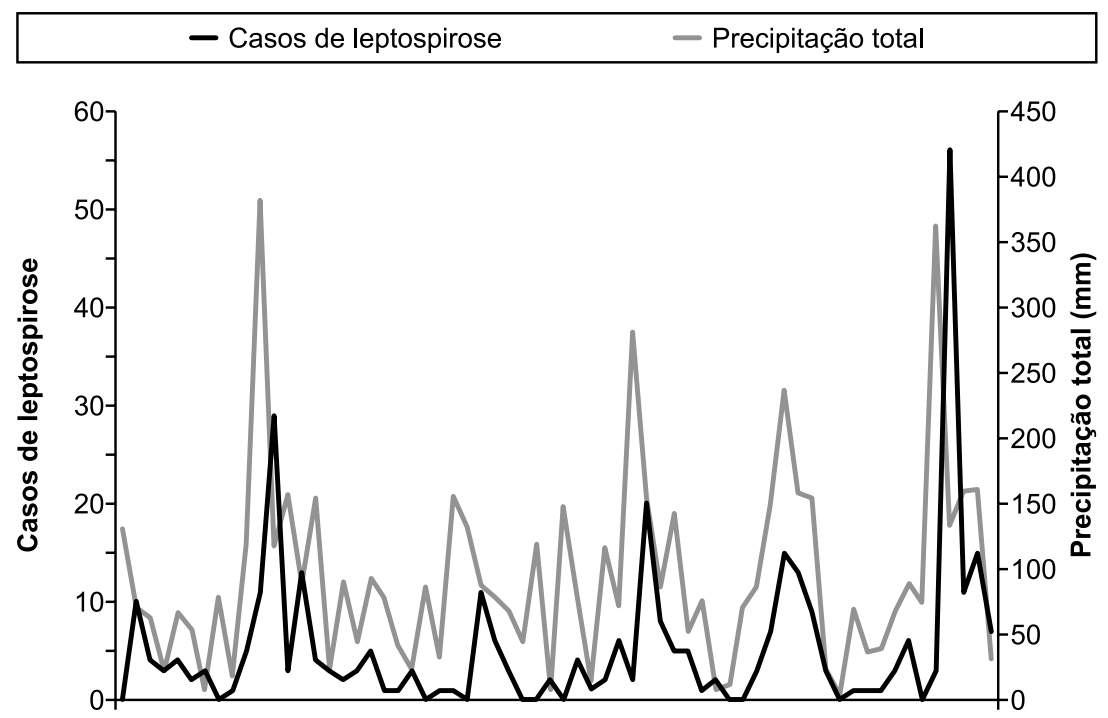

Figura 2. Casos de leptospirose confirmados pelo LACEN e precipitação pluviométrica total.

Mas, se observarmos o número de óbitos, constataremos que ocorreram mais óbitos em Santa Catarina nos anos de 1992, 1993 e 1995, com letalidade maior em 1992 e 1993 (Tabela 1).

Tabela 1. Casos de leptospirose na Grande Florianópolis (GF) e no Estado de Santa Catarina (SC) nos anos de 1992, 1993, 1994 e 1995

\begin{tabular}{|c|c|c|c|c|c|c|c|c|}
\hline \multirow{2}{*}{ Casos } & \multicolumn{2}{|c|}{1992} & \multicolumn{2}{|c|}{1993} & \multicolumn{2}{|c|}{1994} & \multicolumn{2}{|c|}{1995} \\
\hline & GF & $\mathrm{SC}$ & GF & $\mathrm{SC}$ & GF & $\mathrm{SC}$ & GF & $\mathrm{SC}$ \\
\hline Casos notificados & 97 & 188 & 51 & 137 & 177 & 277 & 294 & 583 \\
\hline Casos investigados & 27 & 98 & 53 & 127 & 164 & 258 & 236 & 479 \\
\hline Casos confirmados & 49 & 104 & 35 & 86 & 96 & 168 & 71 & 191 \\
\hline $\mathrm{N}^{o}$ de óbitos & 06 & 19 & 2 & 14 & 18 & 4 & 4 & 20 \\
\hline Taxa de Letalidade \% & 12,24 & 18,44 & 5,55 & 16,09 & 7,00 & 11,00 & 5,63 & 10,47 \\
\hline
\end{tabular}

(Faversani et al., 1999. SES/SC, 93a, b; 94a, b; 95).

Ora, em 1993, não houve enchente (Figura 1, Figura 2), definindo-se enchente como precipitação acima do esperado no local e na época do ano. 
Se os casos de leptospirose são geralmente associados a enchentes, deve-se esperar um maior número de solicitações de exames para confirmação de diagnóstico nos anos em que elas ocorrem. De fato, em 1993, ano sem enchente, diminuiu o número de exames realizados pelo LACEN, mas a porcentagem de resultados positivos foi semelhante, quando comparada à dos anos de enchente (Tabela 2), o que atesta a sensibilidade dos exames.

Tabela 2. Exames realizados pelo LACEN entre 1991 e 1996.

\begin{tabular}{lccc}
\hline Ano & $\mathrm{N}^{\circ}$ de exames & $\mathrm{N}^{\mathrm{o}}$ de casos positivos & Proporção exames/Casos Positivos \% \\
\hline 1991 & 547 & 71 & 12,98 \\
\hline 1992 & 343 & 39 & 11,37 \\
\hline 1993 & 250 & 30 & 12,00 \\
\hline 1994 & 476 & 59 & 12,39 \\
\hline 1995 & 472 & 55 & 11,65 \\
\hline $1996^{*}$ & 901 & 89 & 9,88 \\
\hline Total & 2.989 & 343 & \\
\hline
\end{tabular}

*até abril. FONTE: SES, 1999a.

Assim, em 1993 foram solicitados menos exames laboratoriais, mas o número de casos foi elevado e a taxa de letalidade foi maior do que em 1994 e 1995.

\section{CONCLUSÕES}

A hipótese aqui apresentada é a de que a coincidência entre as curvas de pluviosidade e casos de leptospirose constitui um artifício estatístico e devese ao fato de que, quando ocorrem enchentes, a possibilidade de leptospirose é imediatamente lembrada e mais exames são solicitados. Nos anos em que não há enchente, o diagnóstico de leptospirose é feito tardiamente, o que pode explicar o aumento do número de óbitos.

\section{ABSTRACT}

Leptospirosis and floods: a false correlation?

It is generally admitted that rats are dispersed during floods and bacteria are disseminated through their urine. A retrospective analysis of human cases of leptospirosis during the period of 1991-1996 in the State of Santa Catarina, and the city of Florianópolis, Brazil, confirmed by serology, correlates the incidence of floods with epidemics. The correlation of floods and outbreaks could be spurious, as suggested by the analysis of the coincidence of these events in that period with the data on cases notified, requests for laboratory tests and mortality.

KEY WORDS: Leptospirosis. Floods. Epidemics. Correlations. 


\section{REFERÊNCIAS}

1. Acha PN, Szyfres B. Zoonosis y enfermedades transmisibles comunes al hombre y a los animales. OPAS/OMS, Washington, D.C. 1986.

2. Barcellos C, Sabrosa PC. The place behind the case: leptospirosis risks and associated environmental conditions in a flood-related outbrake in Rio de Janeiro. Cad Saúde Públ 17(Supl): 59-67, 2001.

3. Bolin C. Leptospirosis Ecuador (2). ProMED mail promed@usa.healthnet.org 23 Feb 1998 http://www.healthnet.org/dist/getweb/help/HELP.html

4. Ciceron L, Stepan E, Pinto A, Pizzocaro P, Dettori G, Franzin L, Lupidi R, Mansueto S, Manera A, Ioli A, Marcuttio L, Grillo R, Ciarrocchi S, Cinco M. Epidemiological trend of human leptospirosis in Italy between 1994 and 1996. Europ J Epidemiol 16: 79-86, 2000.

5. Collares-Pereira M, Korver H, Terpstra W, Santos-Reis M, Ramalhinho M, Mathias M, Oom M, Fons R, Libois R, Petrucci-Fonseca F. First epidemiological data on pathogenic leptospires isolated on the Azorean islands. Europ J Epidemiol 13: 435-441, 1997.

6. Collares-Pereira M, Mathias M, Santos-Reis M, Ramalhinho M, Duarte-Rodrigues P. Rodents and Leptospira transmission in Terceira island (Azores). Europ J Epidemiol 16: 1151-1157, 2000.

7. Faversani MC, .Malherbi VC, Philippi JM. Estudo retrospectivo: casos de leptospirose e precipitação pluviométrica de 1991 a 1996 na Grande Florianópolis. Universidade Federal de Santa Catarina, Programa de Pós Graduação em Saúde Pública. Mimeo, 1999.

8. Kupek E, Faversani MC. The relationship between rainfall and human leptospirosis in Florianópolis, Brazil, 1991-1996. Brazil J Infect Dis 4: 131-134, 2000.

9. Levett, PN. Leptospirosis \& dengue: Barbados. promed@usa.healthnet.org 9 Nov 1995. http://www.healthnet.org/dist/getweb/help/HELP.html

10. Michel V, Ruvoen-Clouet N, Menard A, Sonrier C, Filloneau C, Rakotovao F, Ganière J, AndréFontaine G. Role of the coypu (Myocastor coypu) in the epidemiology of leptospirosis in domestic animals and humans in France. Europ J Epidemiol 17: 111-121, 2001.

11. Pollack MP. Leptospirosis - Thailand.promed@usa.healthnet.org 24 Oct 1999 http://www. healthnet.org/dist/getweb/help/HELP.html 